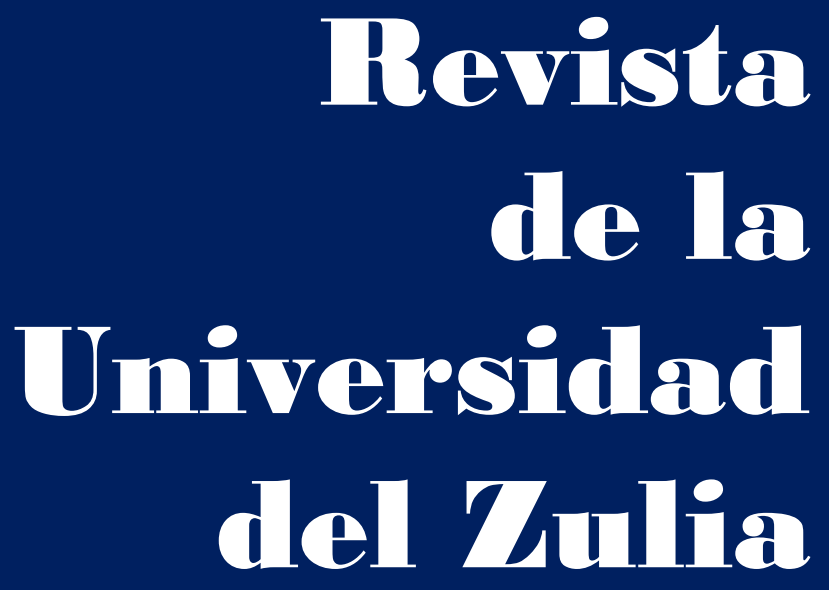

Fundada en 1947

por el Dr. Jesús Enrique Lossada

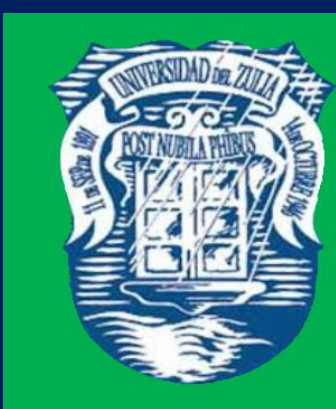

Ciencias del

Agred

Ingemieria

y Teenología
Añต 12 No 32

Enero - Abril 2021

Tercera Época

Maracailbo-Venezuela 


\title{
Atributos de penetración de calor en langostinos procesados térmicamente en envases de vidrio
}

\author{
Diego Alejandro Pulache Celi* \\ William Rolando Miranda Zamora** \\ Luis Manuel Zamora Valladares*** \\ Nelly Luz Leyva Povis****
}

\begin{abstract}
RESUMEN El procesado térmico es una operación vital en el área de la conservación de alimentos. La conserva en vidrio permite extender la durabilidad de los productos. Se estudiaron las características de penetración por calentamiento del producto procesado en retorta por aplicación de vapor de agua. Las conservas de langostino (Litopenaeus vannamei) contenidas en envases de vidrio y en medio de salmuera, se procesó térmicamente hasta valores mínimos de $\mathrm{F}_{0}$ de 5,16 min, 2,61 min, 1,06 min y 0,73 min a $121^{\circ} \mathrm{C}$. Los valores de penetración de calor se registraron para cada minuto de procesamiento con el registrador de temperatura. Se observó que el tiempo para alcanzar la temperatura de retorta $121^{\circ} \mathrm{C}$ osciló entre 19 min a 27 min en vapor de agua. Los parámetros de penetración de calor de las conservas de langostinos en vidrio oscilaron entre $f_{h}$ entre 7,94 a 24,1 min y $j_{h}$ entre 0,66 a 2,65. El tiempo de proceso total pronosticado con el método de Ball modificado osciló entre 19,69 a 57,92 min para las conservas de langostinos en salmuera en vidrio esterilizadas en vapor de agua. Los atributos de penetración de calor, en las conservas de langostinos en salmuera en vidrio, permitieron lograr la esterilidad comercial.
\end{abstract}

PALABRAS CLAVE: Método de Ball modificado, procesamiento térmico, alimentos envasados, cálculos de procesos.

*Estudiante de Ingeniería de Industrias Alimentarias. Universidad Nacional de Frontera, Sullana. Perú. ORCID: https://orcid.org/0000-0001-8582-9763

**Docente Investigador. Universidad Nacional de Frontera, Sullana. Perú. ORCID: https://orcid.org/0000-0002-0829-2568

***Ingeniero Mecatrónico. Universidad Nacional de Piura. Perú. ORCID: https://orcid.org/0000-0002-6067-3552

****Docente Asociada. Universidad Nacional de Piura. Perú. ORCID: https://orcid.org/0000-0003-1821-1044 


\section{Heat penetration attributes in thermally processed prawns in glass containers}

ABSTRACT

Thermal processing is a vital operation in the area of food preservation. The preservation in glass allows to extend the durability of the products. The characteristics of penetration by heating of the processed product in retort by the application of steam were studied. The canned shrimp (Litopenaeus vannamei) contained in glass containers and in a brine medium were thermally processed to minimum values of $F_{0}$ of $5.16 \mathrm{~min}, 2.61 \mathrm{~min}, 1.06 \mathrm{~min}$ and 0.73 $\min$ at $121^{\circ} \mathrm{C}$. The heat penetration values were recorded for each minute of processing with the temperature recorder. It was observed that the time to reach the retort temperature $121^{\circ} \mathrm{C}$ ranged from $19 \mathrm{~min}$ to $27 \mathrm{~min}$ in steam. The parameters of heat penetration of the canned prawns in glass ranged from $\mathrm{f}_{\mathrm{h}}$ between 7.94 to $24.1 \mathrm{~min}$ and $\mathrm{j}_{\mathrm{h}}$ between 0.66 to 2.65. The total process time predicted with the modified Ball method ranged from 19.69 to 57.92 min for canned prawns in glass brines sterilized in steam. The heat penetration attributes of canned prawns in glass brine, allowed achieving commercial sterility.

KEYWORDS: Modified Ball method, thermal processing, canned foods, process calculations.

Introducción

El procesamiento térmico se encuentra entre los primeros métodos de procesamiento comercial (Wang et al., 2019; Membré \& van Zuijlen, 2011; Augusto et al., 2014; Ozilgen, 2011). El tiempo de procesamiento o procesado térmico requerido para reducir la carga microbiana inicial $\mathrm{N}_{0}$, a una concentración final segura $\mathrm{N}$, a una temperatura constante $\mathrm{T}_{\text {ref }}$ (Gao et al., 2020; Nunes et al., 1993) es:

$$
F_{\text {requerido }}=D_{\text {Tref }} \log \left(\frac{N_{0}}{N}\right)
$$

donde $\log \left(\mathrm{N}_{0} / \mathrm{N}\right)$ es el número de reducciones de ciclos logarítmicos microbianos necesarios para lograr un producto seguro (Rifna et al., 2019; Li et al., 2019; Dilmaçünal \& Kuleaşan, 2018; Alexandre et al., 2011) y $D_{\text {Tref }}$ es el tiempo de calentamiento a la $T_{\text {ref }}$ para la reducción de un ciclo logarítmico (Abe et al.,2018; Rahman et al., 2004). 
REVISTA DE LA UNIVERSIDAD DEL ZULIA. $3^{a}$ época. Año 12 N 32, 2021

Diego Alejandro Pulache Celi et al. // Atributos de penetración de calor en langostinos... 114-126

DOI: http://dx.doi.org/10.46925//rdluz.32.09

El procesamiento térmico recibido a temperatura variable $\mathrm{T}(\mathrm{t})$, puede calcularse como:

$$
F_{\text {proceso }}=\int_{0}^{t} 10^{\frac{T-T r e f}{z_{\text {microorgaismo }}}} d t
$$

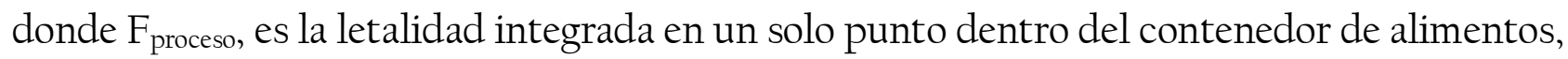
también conocido como valor de esterilización. También se puede definir como el tiempo equivalente de esterilización a una temperatura determinada $\left(\mathrm{T}_{\text {ref }}\right)$ expresada en minutos, $\mathrm{t}$ es la duración del proceso, $z$, es el valor de resistencia del microorganismo de referencia al analizar la cinética de muerte microbiana, o la cantidad de grados Celsius, necesarios para reducir D, en un factor de 10 (Zhang et al., 2018; Dumitraşcu et al., 2012). Las esporas de Clostridium botulinum se han utilizado tradicionalmente, para validar la seguridad de los alimentos de baja acidez esterilizados térmicamente (Tola \& Ramaswamy, 2018; Daryaei et al., 2016; Hong et al., 2016; Johnson, 2014; Taylor et al., 2013; Glass \& Marshall, 2013; Silva \& Gibbs, 2010; Lindström et al., 2006). El centro de la lata se considera crítico; es decir, el último punto esterilizado del alimento calentado (Barbosa-Canovas \& Ibarz, 2014). Por lo tanto, el lado derecho de la ecuación [2], generalmente se evalúa en el punto crítico del alimento. El procesamiento recibido por los alimentos se considera seguro cuando:

$$
F_{\text {proceso }} \geq F_{\text {requerido }}
$$

De la misma manera el valor C (cocción) de un proceso, se define en términos de los parámetros cinéticos, asociados con la pérdida de nutrientes, o el ablandamiento del tejido en el procesamiento térmico:

$$
C_{\text {proceso }}=\int_{0}^{t} 10^{\frac{T-T r e f}{z_{\text {nutriente }}}} d t
$$

donde $z_{\text {nutriente }}$ (valor z) y $\mathrm{T}_{\text {ref }}$ (temperatura de referencia), representan el componente más inestable al calor. En general, el valor de cocción de referencia se caracteriza por: $Z_{\text {nutriente }}=$ $33^{\circ} \mathrm{C}, \mathrm{y} \mathrm{T}_{\text {ref }}=100^{\circ} \mathrm{C}$ (Yang et al., 2016; Holdsworth \& Simpson, 2015a; Tribuzi et al., 2015; Ling et al., 2014; Abakarov \& Nuñez, 2013; Bignardi et al., 2013; IFT/FDA, 2000; Kyereme et al., 
1999). Los valores de F y C, se informan juntos para describir el efecto del procesamiento sobre la carga microbiana, y el valor nutricional de los alimentos (Adepoju et al., 2016). La evaluación del proceso térmico de un alimento se puede hacer usando el método de la Fórmula de Ball (Holdsworth \& Simpson, 2015b; Stoforos, 2010; Stoforos et al., 1997; Stoforos, 1991; Ball, 1928), que hace uso de los parámetros de penetración de calor (Holdsworth \& Simpson, 2015c; Datta, 1990). El método de Ball modificado (MirandaZamora et al., 2020a) usa la siguiente expresión matemática en la parte de la hipérbola planteada por Ball (1923):

$$
\mathrm{C}_{\mathrm{cl}}=\left[\frac{b_{c}}{a_{c} m} \frac{\sqrt{\left.\left(\mathrm{a}_{\mathrm{c}} m+0,343 m\right)^{2}-\left(a_{c} m\right)^{2}\right]}+\frac{b_{c} \ln 10 \cdot \mathrm{z}_{\mathrm{e}} \mathrm{e}^{\frac{\mathrm{a}_{\mathrm{c}} \mathrm{m}}{z_{\mathrm{c}}}}}{a_{c}} \mathrm{~m}}{\mathrm{e}^{\frac{0,343 \mathrm{~m}}{z_{\mathrm{e}}}}} \mathrm{E}\right]
$$

donde $C_{\mathrm{cl}}=$ es la contribuciones del valor $C$ de la parte curva del inicio del enfriamiento, $a_{c} y$ $b_{c}=$ son los coeficientes del modelo de Ball, $m=$ es la diferencia de temperatura entre $T_{g}=l a$ temperatura máxima de calentamiento y $\mathrm{T}_{\mathrm{CW}}$ = la temperatura del agua de enfriamiento, $z_{\mathrm{e}}=$ es el valor $z$ dividido entre el $\ln 10$, y $\mathrm{E}$ = es la integral de la parte curva del modelo de Ball.

El objetivo del trabajo de investigación ha sido establecer los atributos de penetración de calor en langostinos procesados térmicamente en envases de vidrio, para garantizar la esterilidad comercial de las conservas elaboradas determinando los tiempos de proceso total.

\section{Materiales y métodos}

La conserva de langostinos en medio de salmuera ha sido elaborada siguiendo la guía de inspecciones de alimentos enlatados de baja acidez de la FDA (2014). Los langostinos fueron extraídos de una empresa (langostinera) de la ciudad de Tumbes (al norte del Perú). Los envases de vidrio usados fueron de $212 \mathrm{~mL}$ de capacidad, colocándose en su interior 12 langostinos. Las pruebas se realizaron con cuatro réplicas. La evaluación de las pruebas realizadas en conservas de langostino en vidrio, se realizó mediante el método de Ball modificado (Miranda-Zamora et al., 2020a; Tucker et al., 2020), para lo cual se determinó el tiempo de levante o de alcance de la temperatura de retorta (CUT, min), la temperatura inicial $\left(\mathrm{T}_{0},{ }^{\circ} \mathrm{C}\right)$, el tiempo de proceso total $\left(\mathrm{T}_{\mathrm{P}}\right.$, min) y el valor $\mathrm{F}$, del proceso $(\mathrm{min})$ a partir de 
las cuatro pruebas experimentales realizadas en una retorta o autoclave con vapor de agua usando sensores de temperatura. Los datos de las pruebas de penetración de calor fueron obtenidos mediante sensores o termocuplas tipo K (Liu et al., 2020). Los parámetros de penetración de calor del método de Ball modificado $\mathrm{f}_{\mathrm{h}}(\mathrm{min})$ y $\mathrm{j}_{\mathrm{h}}$ fueron determinados usando el algoritmo de un programa realizado en lenguaje Visual (Miranda-Zamora et al., 2020b) a partir del modelo de la ecuación [5] pudiéndose establecer del historial tiempotemperatura, el valor $\mathrm{C}$ del proceso y el tiempo de Ball (B). La temperatura de procesado térmico de las conservas fue de $121^{\circ} \mathrm{C}$ de temperatura y $14,7 \mathrm{psi}\left(\mathrm{lbf} / \mathrm{pulg}^{2}\right)$

\section{Resultados y discusión}

Los resultados del efecto de la esterilización en vapor de agua, en los atributos de penetración de calor del langostino procesado en medio de salmuera, y envasado en vidrio se presenta en la Tabla l. Los gráficos que muestran el valor $\mathrm{F}_{0}$, el valor de cocción (C), la temperatura de la retorta, y la temperatura del producto se dan en las Figuras 1 a la 4.

Tabla l. Factores de penetración de calor y tiempos de proceso total y de Ball por el método de Ball modificado.

\begin{tabular}{|c|c|c|c|c|c|c|c|}
\hline \multirow[t]{2}{*}{ Prueba } & \multirow{2}{*}{$\begin{array}{l}\text { Valor C, proceso } \\
\text { (min) }\end{array}$} & \multirow{2}{*}{$\begin{array}{l}\text { CUT }^{1} \\
(\min )\end{array}$} & \multirow{2}{*}{$\begin{array}{c}\mathrm{f}_{\mathrm{h}}^{2} \\
(\mathrm{~min})\end{array}$} & \multicolumn{2}{|c|}{$\mathrm{j}_{\mathrm{h}}{ }^{2}$} & \multirow{2}{*}{$\begin{array}{c}\mathrm{B}^{3} \\
(\mathrm{~min})\end{array}$} & \multirow{2}{*}{$\begin{array}{l}\mathrm{T}_{\mathrm{P}}{ }^{4} \\
(\mathrm{~min})\end{array}$} \\
\hline & & & & $\begin{array}{l}\text { Con } \\
\text { CUT }\end{array}$ & $\begin{array}{l}\text { Sin } \\
\text { CUT }\end{array}$ & & \\
\hline I & 66,70 & 27 & 24,1 & 1,58 & 7,06 & 42,25 & 57,92 \\
\hline II & 35,90 & 20 & 17,11 & 0,76 & 3,61 & 22,42 & 34 \\
\hline III & 16,84 & 23 & 7,94 & 2,65 & 126,68 & 14,31 & 27,64 \\
\hline IV & 15,25 & 19 & 8,23 & 0,66 & 14,36 & 8,69 & 19,69 \\
\hline
\end{tabular}

${ }^{\mathrm{l} C U T}=$ tiempo de levante de la temperatura de la retorta $\mathrm{u}$ autoclave; ${ }^{2} \mathrm{f}_{\mathrm{h}} \mathrm{y} \mathrm{j}_{\mathrm{h}}=$ párametros de penetración del calor; ${ }^{3} \mathrm{~B}=$ tiempo de $\mathrm{Ball} ;{ }^{4} \mathrm{~T}_{\mathrm{P}}=$ tiempo de proceso total o calentamiento) $=\mathrm{B}$ $+0,58$ CUT.

El tiempo de proceso o tiempo del operador, $t_{\mathrm{op}}$, para una operación comercial se mide desde el momento en que la retorta alcanza la temperatura de procesamiento o de retorta, $T_{R}$, hasta el momento en que se apaga el vapor, y se aplica el agua de enfriamiento. Sin embargo, hay un tiempo significativo para que la retorta alcance la temperatura de procesamiento, o de retorta, lo que contribuye al efecto letal total; esto se conoce como el 
"tiempo de levante" o CUT (Terajima, 2016; Simpson et al., 2012; Berry, 1983; Succar \& Kayakawa, 1982; Ikegami, 1974). El CUT describe el tiempo que tardó la retorta en alcanzar la temperatura requerida de $121^{\circ} \mathrm{C}$ para que fuera disminuyendo en la aplicación de vapor de agua a los 27, 20, 23 y 19 minutos para el langostino en medio de salmuera y envase de vidrio conforme se desea alcanzar el valor $\mathrm{F}$ requerido de $5 \mathrm{~min}, 2,52 \mathrm{~min}, 1 \mathrm{~min}$ y 0,5 $\mathrm{min}$ respectivamente. En el procesamiento de retorta, el tiempo que tarda una curva de penetración de calor en atravesar un ciclo logarítmico se denomina valor de índice de velocidad de calentamiento, $\mathrm{f}_{\mathrm{h}}$. El índice de velocidad de calentamiento $\left(\mathrm{f}_{\mathrm{h}}\right)$ para el calentamiento por convección, es un factor que depende de la temperatura del medio de calentamiento, así como de la superficie de condensación en términos de tamaño, y orientación de la superficie. Esto explica el valor $f_{h}$ en el langostino en medio de salmuera envasado en vidrio procesado en retorta con vapor de agua como medio de calentamiento (Tabla 1).

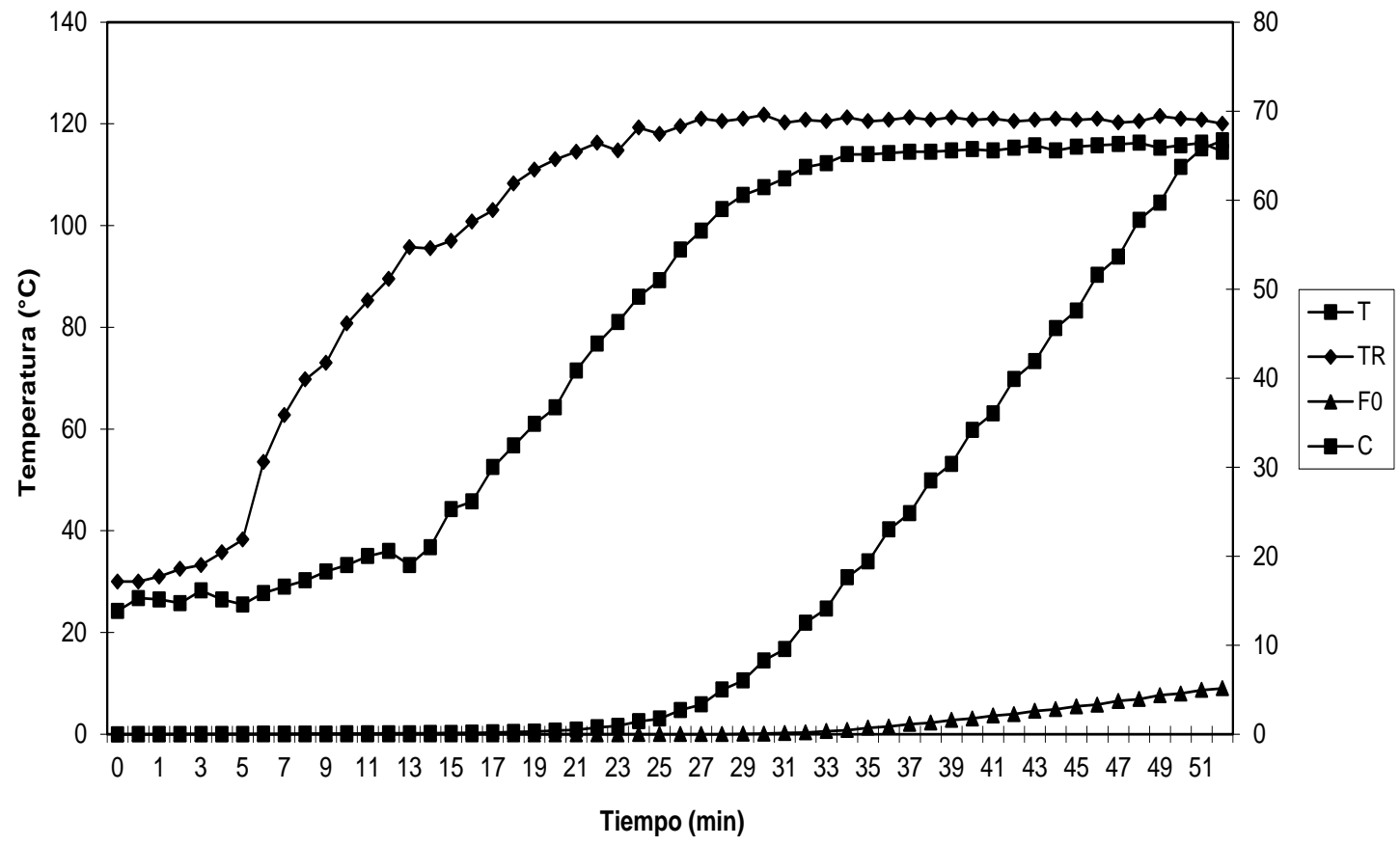

Figura 1. Atributos de penetración de calor (valor $\mathrm{F}_{0}$, valor de cocción, temperatura de retorta y temperatura central) del langostino procesado térmicamente por vapor de agua en un envase de vidrio basado en la temperatura $\left({ }^{\circ} \mathrm{C}\right)$ y el tiempo (minutos). La temperatura interna al comienzo y al final del procesamiento $=24,25^{\circ} \mathrm{C}$ y $114,5^{\circ} \mathrm{C}$, respectivamente. La temperatura de retorta al comienzo y al final del procesamiento $=30^{\circ} \mathrm{C}$ y $120^{\circ} \mathrm{C}$, respectivamente. La letalidad $\left(F_{0}\right)$ 5,16 y el valor de cocción 66,70 se lograron a los 52 min. 


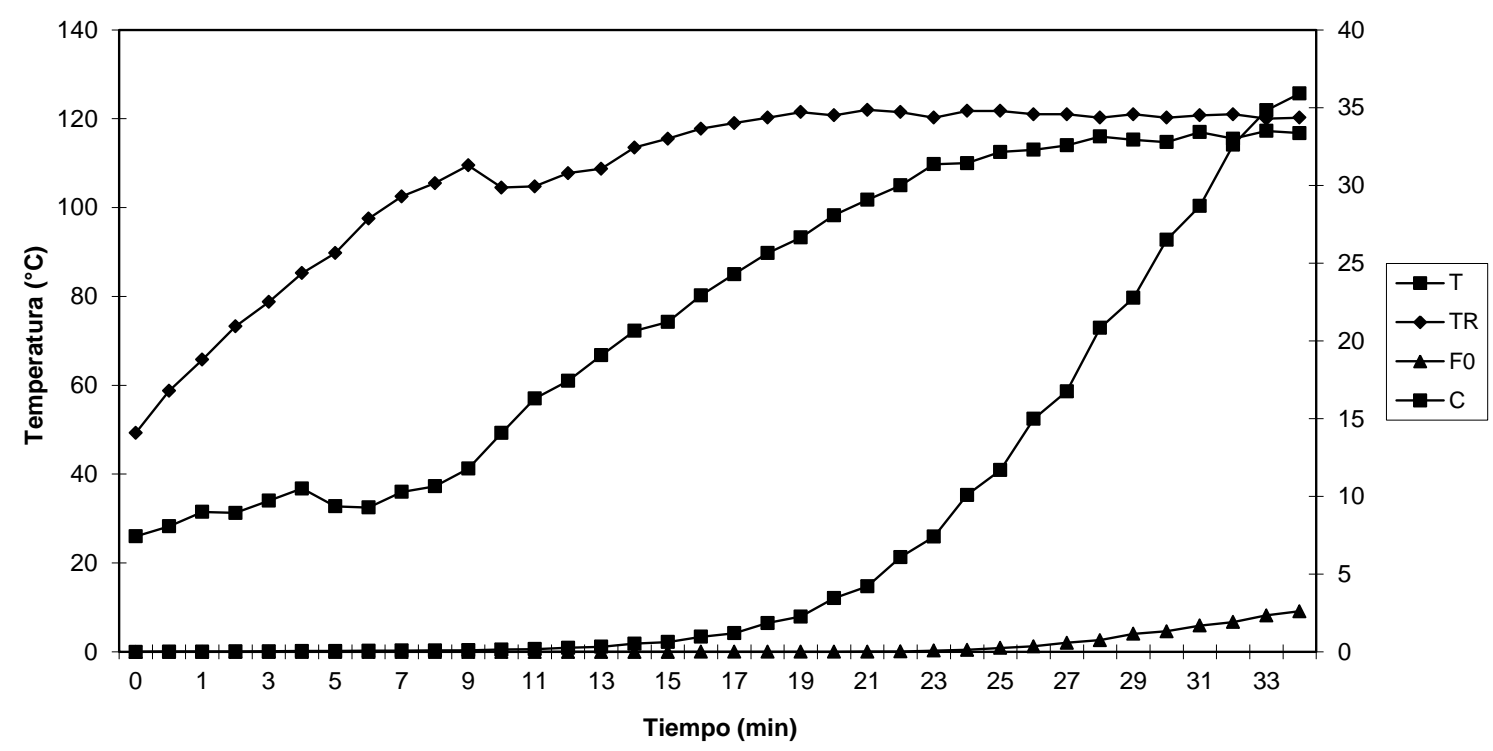

Figura 2. Atributos de penetración de calor (Valor $F_{0}(F 0)$, valor de cocción (C), temperatura de retorta (TR) y temperatura central (T)) del langostino procesado térmicamente por vapor de agua en un envase de vidrio basado en la temperatura $\left({ }^{\circ} \mathrm{C}\right)$ y el tiempo (minutos). La temperatura interna al comienzo y al final del procesamiento $=26^{\circ} \mathrm{C}$ y $116,75^{\circ} \mathrm{C}$, respectivamente. La temperatura de retorta al comienzo y al final del procesamiento $=49,25^{\circ} \mathrm{C}$ y $120,25^{\circ} \mathrm{C}$, respectivamente. La letalidad $\left(\mathrm{F}_{0}\right)$ 2,61 y el valor de cocción 35,90 se lograron a los $34 \mathrm{~min}$.

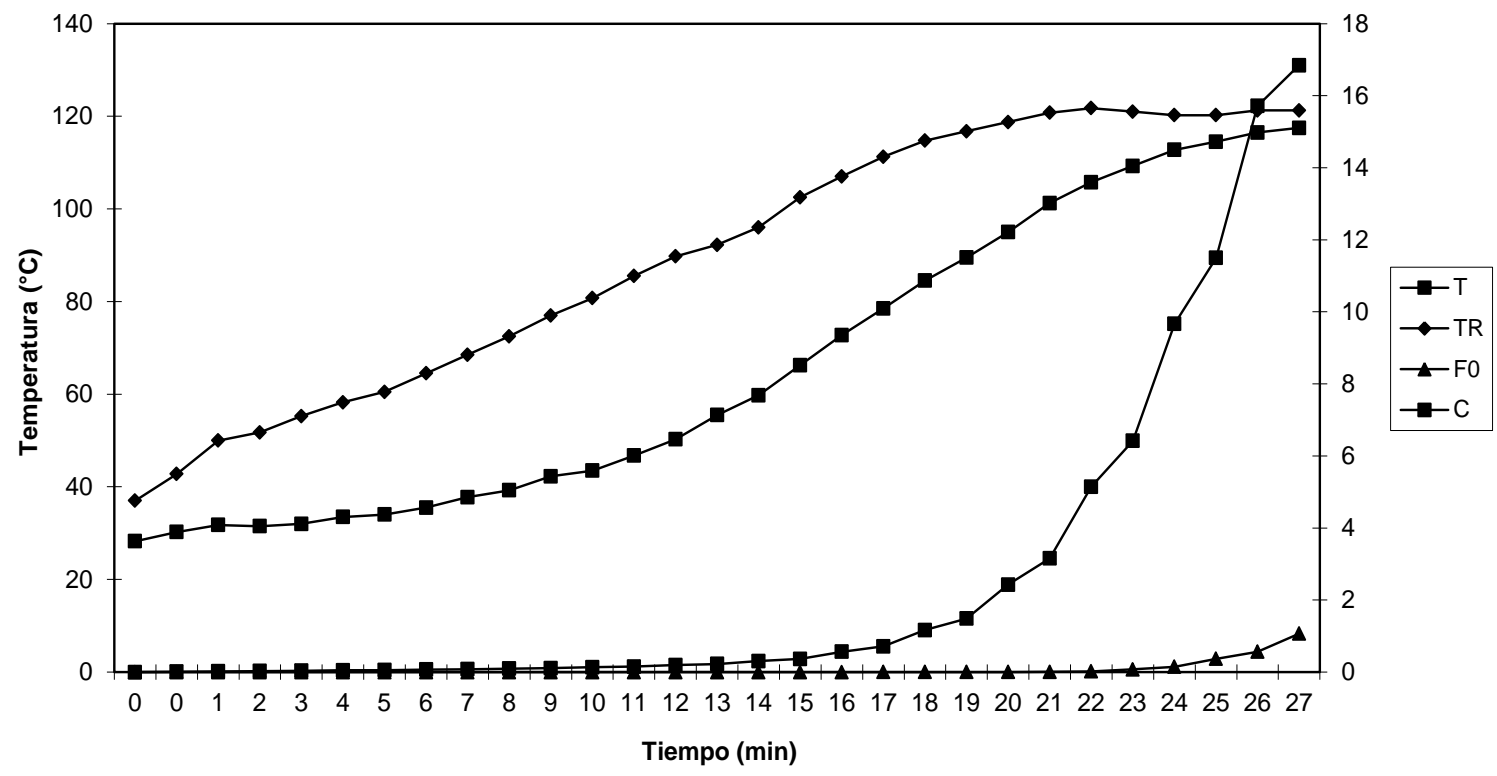

Figura 3. Atributos de penetración de calor (Valor $F_{0}(F 0)$, valor de cocción (C), temperatura de retorta (TR) y temperatura central (T)) del langostino procesado térmicamente por vapor de agua en un envase de vidrio basado en la temperatura $\left({ }^{\circ} \mathrm{C}\right.$ ) y el tiempo (minutos). La temperatura interna al comienzo y al final del procesamiento $=28,25^{\circ} \mathrm{C}$ y $117,5^{\circ} \mathrm{C}$, respectivamente. La temperatura de retorta al comienzo y al final del procesamiento $=37^{\circ} \mathrm{C}$ y $121,25^{\circ} \mathrm{C}$, respectivamente. La letalidad $\left(\mathrm{F}_{0}\right)$ 1,06 y el valor de cocción 16,84 se lograron a los 27 $\min$. 


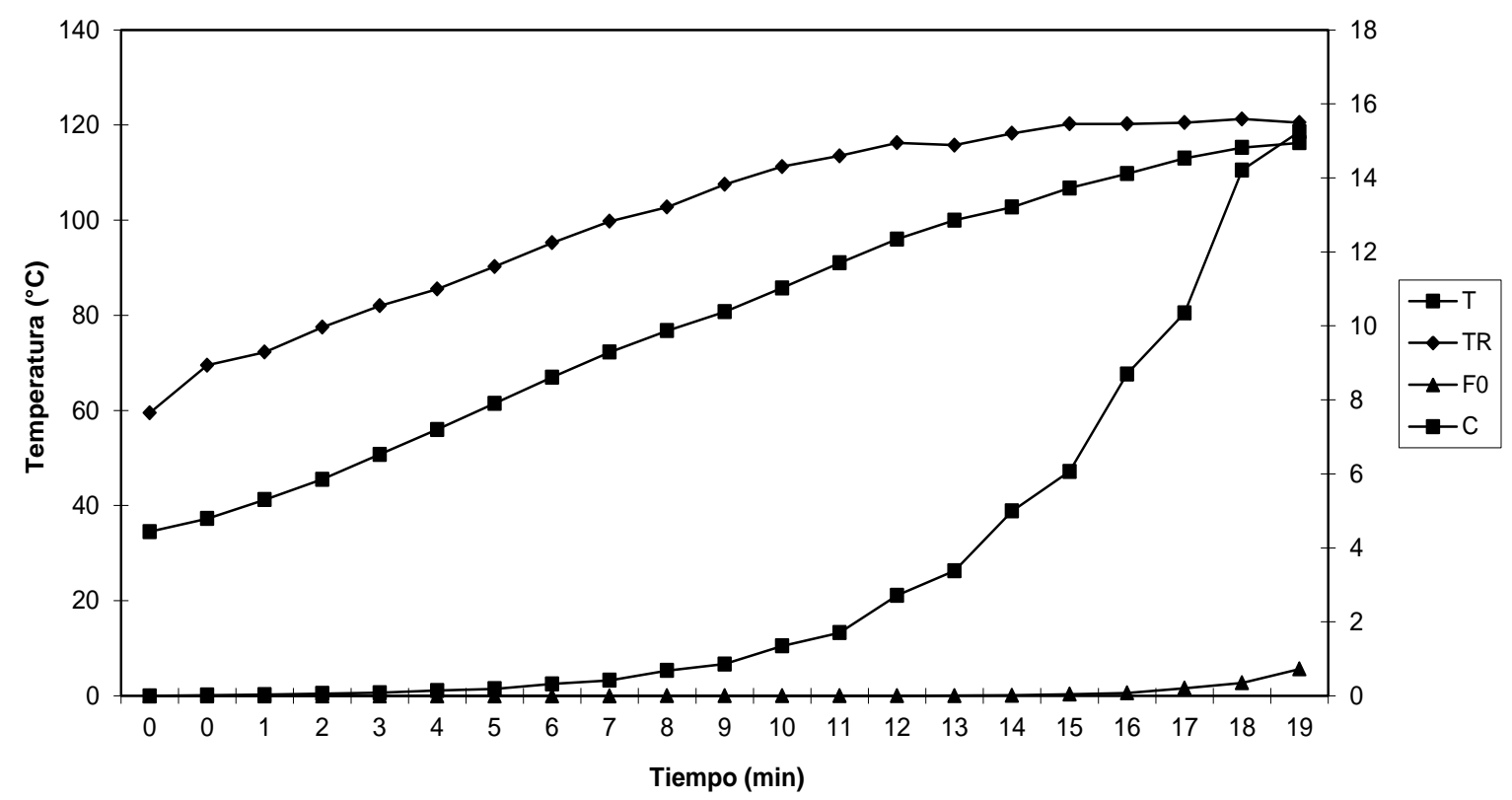

Figura 4. Gráfico que muestra los atributos de penetración de calor (Valor $F_{0}(F 0)$, valor de cocción (C), temperatura de retorta (TR) y temperatura central (T)) del langostino procesado térmicamente por vapor de agua en un envase de vidrio basado en la temperatura $\left({ }^{\circ} \mathrm{C}\right)$ y el tiempo (minutos). La temperatura interna al comienzo y al final del procesamiento $=34,5^{\circ} \mathrm{C}$ y $116,25^{\circ} \mathrm{C}$, respectivamente. La temperatura de retorta al comienzo y al final del procesamiento $=59,5^{\circ} \mathrm{C}$ y $120,5^{\circ} \mathrm{C}$, respectivamente. La letalidad $\left(\mathrm{F}_{0}\right) 0,73$ y el valor de cocción 15,25 se lograron a los $19 \mathrm{~min}$.

En los alimentos procesados en latas, el factor de retraso o demora para el calentamiento $\left(\mathrm{j}_{\mathrm{h}}\right.$ ) está relacionado con el tiempo de retraso requerido para alcanzar valores de velocidad de calentamiento armonizados (Mohan et al., 2015). El factor de retraso de calentamiento $\left(\mathrm{j}_{\mathrm{h}}\right.$ ) cercano o superior a 1,0, es una indicación de una penetración de calor más rápida y esto es evidente en las pruebas I y III $\mathrm{j}_{\mathrm{h}}=1,58$, y jh $=2,65$ respectivamente, II y IV con valores por debajo de $1 \mathrm{j}_{\mathrm{h}}=0,76 \mathrm{y} \mathrm{j}_{\mathrm{h}}=0,66$ respectivamente. El factor de retraso de calentamiento $\left(\mathrm{j}_{\mathrm{h}}\right)$ en dos de los procesos fue superior a 1 , y en dos debajo a l. Se observaron valores superiores a 1 de $\mathrm{j}_{\mathrm{h}}$ de 1,444 y 1,445 en camarón kuruma procesados en latas, y cercanos a 1,0 de 0,962 0,8735 para camarones kuruma procesadas en bolsa retortables, respectivamente (Mohan et al., 2008). El valor de cocción (C) se refiere al valor que sugiere el impacto del procesamiento térmico en los alimentos con respecto a la degradación de nutrientes y debe ser lo más mínimo posible en cualquier letalidad dada (Majumdar et al., 2016; Mohan et al., 2015). En este estudio, se observó el C mínimo, en la aplicación de vapor de agua con 15,25 min, 16,84 min, 35,90 min y 66,70 min en los procesos que se aplica 
REVISTA DE LA UNIVERSIDAD DEL ZULIA. 3ª época. Año 12 N 32, 2021

Diego Alejandro Pulache Celi et al. // Atributos de penetración de calor en langostinos... 114-126

DOI: http://dx.doi.org/10.46925//rdluz.32.09

valores $\mathrm{F}_{0}$ de 0,73 $\mathrm{min}, 1,06 \mathrm{~min}, 2,61 \mathrm{~min}$ y 5,16 min, respectivamente. Se observó que el tiempo total del proceso $\left(T_{\mathrm{P}}\right)$ experimental para el langostino en salmuera en envase de vidrio, procesado en vapor de agua fue de $52 \mathrm{~min}, 34 \mathrm{~min}, 27 \mathrm{~min}$ y 19 min para alcanzar un valor $F_{0}$ de proceso de 5,16 min, 2,61 min, 1,06 min y,0,73 min respectivamente. El menor tiempo de proceso observado de $10 \mathrm{~min}$, se logró en la prueba que tuvo el menor valor $\mathrm{F}_{0}$ de proceso, lo que resultó en una velocidad de calentamiento más rápida (Mohan et al., 2015). También se puede observar en la Tabla l, que el método de Ball modificado predice bastante bien los tiempos de proceso total 57,92 min, $34 \mathrm{~min}, 27,64 \mathrm{~min}$ y 19,69 min, para procesos en los que el tiempo experimental fue de $52 \mathrm{~min}, 34 \mathrm{~min}, 27 \mathrm{~min}$ y $19 \mathrm{~min}$, respectivamente.

\section{Conclusiones}

Se establecieron los atributos de penetración de calor (el tiempo de alcance de la temperatura de retorta $u$ autoclave, el valor de cocción, el tiempo de proceso total pronosticado con el método de Ball) en las conservas de langostinos en salmuera en vidrio logrando la esterilidad comercial de las mismas.

\section{Referencias}

Abakarov, A.; Nuñez, M. 2013. Thermal food processing optimization: Algorithms and software. Journal of Food Engineering, 115(4), 428-442.

Abe, H.; Koyama, K.; Kawamura, S.; Koseki, S. 2018. Stochastic evaluation of Salmonella enterica lethality during thermal inactivation. International Journal of Food Microbiology, 285, 129-135.

Adepoju, M.A.; Omitoyin, B.O.; Mohan, C.O.; Zynudheen, A.A.2016. Heat penetration attributes of milkfish (Chanos chanos) thermal processed in flexible pouches: a comparative study between steam application and water immersion. Food Science \& Nutrition, 5(3), 521-524.

Alexandre, E.M.C.; Brandão, T.R.S.; Silva, C.L.M. 201l. Modelling microbial load reduction in foods due to ozone impact. Procedia Food Science, 1, 836-841.

Augusto, P.E.D.; Tribst, A.A.L.; Cristianini, M. 2014. Thermal processes $\mid$ Commercial sterility (Retort). Encyclopedia of Food Microbiology, 567-576.

Ball, C.O. 1923. Thermal process time for canned food. Bulletin of the National Research Council, Washington, DC, 7 Part 1, Number 37. 
REVISTA DE LA UNIVERSIDAD DEL ZULIA. 3ª época. Año 12 Nº 32, 2021

Diego Alejandro Pulache Celi et al. // Atributos de penetración de calor en langostinos... 114-126

DOI: http://dx.doi.org/10.46925//rdluz.32.09

Ball, C.O. 1928. Mathematical solution of problems on thermal processing of canned food. Univ Calif Publ Public Health 1(2):145-245.

Barbosa-Canovas, G.V.; Ibarz, A. 2014. Introduction to food process engineering. CRC Press.

Berry, M.R. 1983. Prediction of come-up time correction factors for batch-type agitating and still retorts and the influence on thermal process calculations. Journal of Food Science, 48(4), 1293-1299.

Bignardi, C.; Cavazza, A.; Rinaldi, M.; Corradini, C.; Massini, R. 2013. Evaluation of thermal treatment markers in wheat flour-derived products cooked in conventional and in lowemissivity ovens. Food Chemistry, 140(4), 748-754.

Daryaei, H.; Balasubramaniam, V. M.; Yousef, A.E.; Legan, J.D.; Tay, A. 2016. Lethality enhancement of pressure-assisted thermal processing against Bacillus amyloliquefaciens spores in low-acid media using antimicrobial compounds. Food Control, 59, 234-242.

Datta, A.K. 1990. On the theoretical basis of the asymptotic semilogarithmic heat penetration curves used in food processing. Journal of Food Engineering, 12(3), 177-190.

Dilmaçünal, T.; Kuleaşan, H. 2018. Novel strategies for the reduction of microbial degradation of foods. Food Safety and Preservation, 481-520.

Dumitraşcu, L.; Stănciuc, N.; Stanciu, S.; Râpeanu, G. 2012. Thermal inactivation of lactoperoxidase in goat, sheep and bovine milk - A comparative kinetic and thermodynamic study. Journal of Food Engineering, 113(1), 47-52.

FDA. 2014. Guide to inspections of low acid canned food 5. Disponible en: https://www.fda.gov/inspections-compliance-enforcement-and-criminalinvestigations/inspection-guides/guide-inspections-low-acid-canned-food-5-0.

Gao, T.; Tian, Y.; Zhu, Z.; Sun, D.-W. 2020. Modelling, responses and applications of timetemperature indicators (TTIs) in monitoring fresh food quality. Trends in Food Science \& Technology, 99, 311-322.

Glass, K.; Marshall, K. 2013. Clostridium botulinum. Foodborne Infections and Intoxications, 371-387.

Holdsworth, S.D.; Simpson, R. 2015a. Optimization of Thermal Food Processing. Thermal Processing of Packaged Foods, 383-414.

Holdsworth, S.D.; Simpson, R. 2015b. Formula methods and analytical techniques. Thermal Processing of Packaged Foods, 219-250.

Holdsworth, S.D.; Simpson, R. 2015c. Heat Penetration in packaged foods. thermal processing of packaged foods, 161-195. 
REVISTA DE LA UNIVERSIDAD DEL ZULIA. 3ª época. Año 12 Nº 32, 2021

Diego Alejandro Pulache Celi et al. // Atributos de penetración de calor en langostinos... 114-126

DOI: http://dx.doi.org/10.46925//rdluz.32.09

Hong, Y.-K.; Huang, L.; Yoon, W. B.; Liu, F.; Tang, J. 2016. Mathematical modeling and Monte Carlo simulation of thermal inactivation of non-proteolytic Clostridium botulinum spores during continuous microwave-assisted pasteurization. Journal of Food Engineering, $190,61-71$.

IFT/FDA. 2000. Kinetics of microbial inactivation for alternative food processing technologies. Journal of Food Science, 65, s8.

Ikegami, Y. 1974. Effect of various factors in the come-up time on processing of canned foods with steam. Report of Toyo Institute of Food Technology (in Japanese), 11, 92-98.

Johnson, E.A. 2014. Clostridium | Clostridium botulinum. Encyclopedia of Food Microbiology, 458-462.

Kyereme, M.; Swartzel, K.R.; Farkas, B.E. 1999. New line intersection procedure for the equivalent point method of thermal evaluation. Journal of Food Science, 64(4), 565-570.

Li, S.; Zhilyaev, S.; Gallagher, D.; Subbiah, J.; Dvorak, B. 2019. Sustainability of safe foods: Joint environmental, economic and microbial load reduction assessment of antimicrobial systems in U.S. beef processing. Science of The Total Environment, 691, 252-262.

Lindström, M.; Kiviniemi, K.; Korkeala, H. 2006. Hazard and control of group II (nonproteolytic) Clostridium botulinum in modern food processing. International Journal of Food Microbiology, 108(1), 92-104.

Ling, B.; Tang, J.; Kong, F.; Mitcham, E.J.; Wang, S. 2014. Kinetics of food quality changes during thermal processing: a Review. Food and Bioprocess Technology, 8(2), 343-358.

Liu, B.; Huang, Q.; Wang, P. 2020. Influence of surrounding gas temperature on thermocouple measurement. Case Studies in Thermal Engineering, 19, 100627.

Majumdar, R.K.; Roy, D.; Saha, A. 2016. Textural and sensory characteristics of retortprocessed freshwater prawn (Macrobrachium rosenbergii) in curry medium. International Journal of Food Properties, 20(11), 2487-2498.

Membré, J.-M.; van Zuijlen, A. 2011. A probabilistic approach to determine thermal process setting parameters: Application for commercial sterility of products. International Journal of Food Microbiology, 144(3), 413-420.

Miranda-Zamora, W.R.; Sanchez-Chero, M.J.; Sanchez-Chero, J.A. 2020a. Software for the determination of the time and the $f$ value in the thermal processing of packaged foods using the modified ball method. Intelligent human systems integration 2020. Proceedings of the 3rd International Conference on Intelligent Human Systems Integration (IHSI 2020): Integrating People and Intelligent Systems, February 19-21, 2020, Modena, Italy, 498-502.

Miranda-Zamora, W.R.; Villarreyes, S.S.C.; Povis, N.L.L.; Panca, C.M.A.; Morales, M.V.S. 2020b. A new mathematical solution for packaged food thermal processing. Advances in Intelligent Systems and Computing, 2020, 1216 AISC, 383-387 
REVISTA DE LA UNIVERSIDAD DEL ZULIA. 3ª época. Año 12 Nº 32, 2021

Diego Alejandro Pulache Celi et al. // Atributos de penetración de calor en langostinos... 114-126 DOI: http://dx.doi.org/10.46925//rdluz.32.09

Mohan, C.O.; Ravishankar, C.N.; Srinivasa Gopal, T.K.; Bindu, J. 2008. Thermal processing of prawn "kuruma" in retortable pouches and aluminium cans. International Journal of Food Science \& Technology, 43(2), 200-207.

Mohan, C.O.; Remya, S.; Murthy, L.N.; Ravishankar, C.N.; Asok Kumar, K. 2015. Effect of filling medium on cooking time and quality of canned yellowfin tuna (Thunnus albacares). Food Control, 50, 320-327.

Nunes, R.V.; Swartzel, K.R.; Ollis, D.F. 1993. Thermal evaluation of food processes: The role of a reference temperature. Journal of Food Engineering, 20(1), 1-15.

Öilgen, M. 201l. Handbook of food process modeling and statistical quality control: with extensive MATLAB applications. CRC Press.

Rahman, M.S.; Guizani, N.; Al-Ruzeiki, M.H. 2004. D- and Z-values of microflora in tuna mince during moist - and dry-heating. LWT - Food Science and Technology, 37(1), 93-98.

Rifna, E.J.; Singh, S.K.; Chakraborty, S.; Dwivedi, M. 2019. Effect of thermal and nonthermal techniques for microbial safety in food powder: Recent advances. Food Research International, 126, 108654.

Silva, F.V.M.; Gibbs, P.A. 2010. Non-proteolytic Clostridium botulinum spores in low-acid cold-distributed foods and design of pasteurization processes. Trends in Food Science \& Technology, 21(2), 95-105.

Simpson, R.; Almonacid, S.; Nuñez, H.; Urtubia, A.; Teixeira, A.A. 2012. Is there a need for the come-up time correction factor in Ball's formula method? A Critical Analysis. Food Engineering Reviews, 4(2), 107-113.

Stoforos, N.G. 1991. On Ball's formula method for thermal process calculations. Journal of Food Process Engineering, 13(4), 255-268.

Stoforos, N.G. 2010. Thermal process calculations through Ball's original formula method: a critical presentation of the method and simplification of its use through regression equations. Food Engineering Reviews, 2(1), 1-16.

Stoforos, N.G.; Noronha, J.; Hendrickx, M.; Tobback, P.; Hayakawa, K. 1997. A critical analysis of mathematical procedures for the evaluation and design of in-container thermal processes for foods. Critical Reviews in Food Science and Nutrition, 37(5), 4ll-44l.

Succar, J.; Kayakawa, K.-I. 1982. Prediction of time correction factor for come-up heating of packaged liquid food. Journal of Food Science, 47(2), 614-618.

Taylor, R.H.; Dunn, M.L.; Ogden, L.V.; Jefferies, L.K.; Eggett, D.L.; Steele, F. M. 2013. Conditions associated with Clostridium sporogenes growth as a surrogate for Clostridium botulinum in nonthermally processed canned butter. Journal of Dairy Science, 96(5), 27542764. 
REVISTA DE LA UNIVERSIDAD DEL ZULIA. 3ª época. Año 12 N 32, 2021

Diego Alejandro Pulache Celi et al. // Atributos de penetración de calor en langostinos... 114-126 DOI: http://dx.doi.org/10.46925//rdluz.32.09

Terajima, Y. 2016. Prediction of come-up time correction factor for heat processing of food in containers using Come-Up heating medium temperatures. Journal of Food Process Engineering, 40(2), el2361.

Tola, Y.B.; Ramaswamy, H.S. 2018. Novel processing methods: updates on acidified vegetables thermal processing. Current Opinion in Food Science, 23, 64-69.

Tribuzi, G.; de Aragão, G.M.F.; Laurindo, J.B. 2015. Processing of chopped mussel meat in retort pouch. Food Science and Technology, 35(4), 612-619.

Tucker, G.S.; Featherstone, S.; Miranda-Zamora, W.R. 2020. Fundamentos del procesamiento térmico de alimentos. AMV Ediciones.

Wang, J.; Jayaprakasha, G.K.; Patil, B.S. 2019. Chemometric characterization of 30 commercial thermal and cold processed juices using UPLC-QTOF-HR-MS fingerprints. LWT, 114, 108322.

Yang, Y.; Achaerandio, I.; Pujolà, M. 2016. Effect of the intensity of cooking methods on the nutritional and physical properties of potato tubers. Food Chemistry, 197, 1301-1310.

Zhang, S.; Zhang, L.; Lan, R.; Zhou, X.; Kou, X.; Wang, S. 2018. Thermal inactivation of Aspergillus flavus in peanut kernels as influenced by temperature, water activity and heating rate. Food Microbiology, 76, 237-244. 